\title{
Dynamic aspects of electronic predissociation
}

\section{Grønager, Michael; Henriksen, Niels Engholm}

Published in:

Journal of Chemical Physics

Link to article, DOI:

$10.1063 / 1.471089$

Publication date:

1996

Document Version

Publisher's PDF, also known as Version of record

Link back to DTU Orbit

Citation (APA):

Grønager, M., \& Henriksen, N. E. (1996). Dynamic aspects of electronic predissociation. Journal of Chemical Physics, 104(9), 3234-3243. https://doi.org/10.1063/1.471089

\section{General rights}

Copyright and moral rights for the publications made accessible in the public portal are retained by the authors and/or other copyright owners and it is a condition of accessing publications that users recognise and abide by the legal requirements associated with these rights.

- Users may download and print one copy of any publication from the public portal for the purpose of private study or research.

- You may not further distribute the material or use it for any profit-making activity or commercial gain

- You may freely distribute the URL identifying the publication in the public portal

If you believe that this document breaches copyright please contact us providing details, and we will remove access to the work immediately and investigate your claim 


\title{
Dynamic aspects of electronic predissociation
}

\author{
Michael Grønager and Niels Engholm Henriksen \\ Chemistry Department B, Technical University of Denmark, DTU 207, DK-2800 Lyngby, Denmark
}

(Received 15 May 1995; accepted 28 November 1995)

\begin{abstract}
We consider electronic excitation induced with a continuous wave laser to an excited bound state which can predissociate due to a radiationless transition to a dissociative state. The conditions for a separation of the process into the preparation of a vibrational eigenstate which subsequently dissociates due to a radiationless transition are established. We point out that the probability of the radiationless transition can be calculated from a time-dependent nuclear autocorrelation function, an expression which nicely reflects the pictorial aspect of the Franck-Condon principle. (C) 1996 American Institute of Physics. [S0021-9606(96)02909-8]
\end{abstract}

\section{INTRODUCTION}

Photodissociation is normally divided into direct and indirect processes. ${ }^{1}$ Indirect processes implies dissociation via an intermediate energized complex. This case is reminiscent of the traditional scheme for unimolecular reactions. The indirect processes can be further classified according to the mode of dissociation of the energized complex. The principal mechanisms are vibrational and electronic predissociation.

Direct and indirect photodissociation can be treated within the same theoretical framework ${ }^{1,2}$ and the distinction between the two cases might indeed, in some cases, be somewhat artificial. It can nevertheless be profitable to focus on particular scenarios where simplifications-conceptually as well as a computationally - can be obtained.

We focus in this paper on situations where excitation and subsequent dissociation can be separated and we consider this limit in connection with electronic predissociation from a bound to a continuum state.

The objective of this paper is to enhance the insight into the time evolution of the predissociation process in a form which, in part, is complementary to "standard" treatments. We want to characterize the situations where one can evaluate the radiationless transition probabilities in terms of Franck-Condon factors. ${ }^{3,4}$ The possibility of creating nonstationary states by femtosecond excitation implies that such a description does not always apply. One of the (implicit) assumptions behind the use of Franck-Condon factors is that a stationary vibrational state has been created prior to dissociation. Even if excitation is carried out with a continuous wave laser the conditions under which a (quasi-)stationary vibrational state is created must be established. This is one of the main objectives of the present paper.

The dynamic aspects of electronic excitation to a single (isolated) excited electronic state have already been discussed in the literature. ${ }^{5,6}$ The nuclear state created by continuous wave excitation is often termed a Raman wave function $^{6,7}$ and some of the properties of this object have been nicely illustrated. ${ }^{6}$ We need, however, for the present purpose to clarify some additional points concerning the nature of the Raman wave function.

Heller $^{8,9}$ pointed out that the transcription of FranckCondon factors into a time-dependent form can be advanta- geous, computationally and from the point of view of physical insight. Heller was discussing photoabsorption and basically the same idea has subsequently been used, e.g., in connection with vibrational predissociation ${ }^{10}$ and nonadiabatic electron transfer rates. ${ }^{11,12}$ We show here that the same idea is advantageous for electronic predissociation (such a computational approach has, in fact, already been suggested in this case for the evaluation of optical line shapes ${ }^{13}$ ).

We have organized this paper in the following way. In Sec. II we first rederive an expression for the transition probability $^{2}$ based on a perturbation treatment for the field only. We then include the radiationless coupling in the perturbation treatment, and derive an expression for the transition probability in terms of Franck-Condon factors. We get such an expression valid in the continuous wave limit, and an expression valid long time after we have removed the influence of the field. In Sec. III we consider the validity of the approximations and test the derived formulas on a numerical example.

\section{THEORY}

In this paper we consider molecules with the following quantum mechanical properties: Three stationary electronic states; a bound ground state, a bound excited state, a repulsive excited state (see Fig. 1), and a weak coupling between the bound and the repulsive excited states. And finally radiative coupling between the ground state and the bound excited state. We shall in the following refer to the three states as the ground, the bound, and the repulsive state and denote them with the subscripts $g, b$, and $r$. The Hamiltonian for such molecules becomes in the diabatic representation,

$$
\begin{aligned}
\hat{H}= & {\left[\begin{array}{ccc}
T(\hat{p}) & 0 & 0 \\
0 & T(\hat{p}) & 0 \\
0 & 0 & T(\hat{p})
\end{array}\right]+\left[\begin{array}{ccc}
V_{g}(\hat{r}) & 0 & 0 \\
0 & V_{b}(\hat{r}) & C_{b r}(\hat{r}) \\
0 & C_{r b}(\hat{r}) & V_{r}(\hat{r})
\end{array}\right] } \\
& +\left[\begin{array}{ccc}
0 & \hat{R}_{g b}(t) & 0 \\
\hat{R}_{b g}(t) & 0 & 0 \\
0 & 0 & 0
\end{array}\right],
\end{aligned}
$$




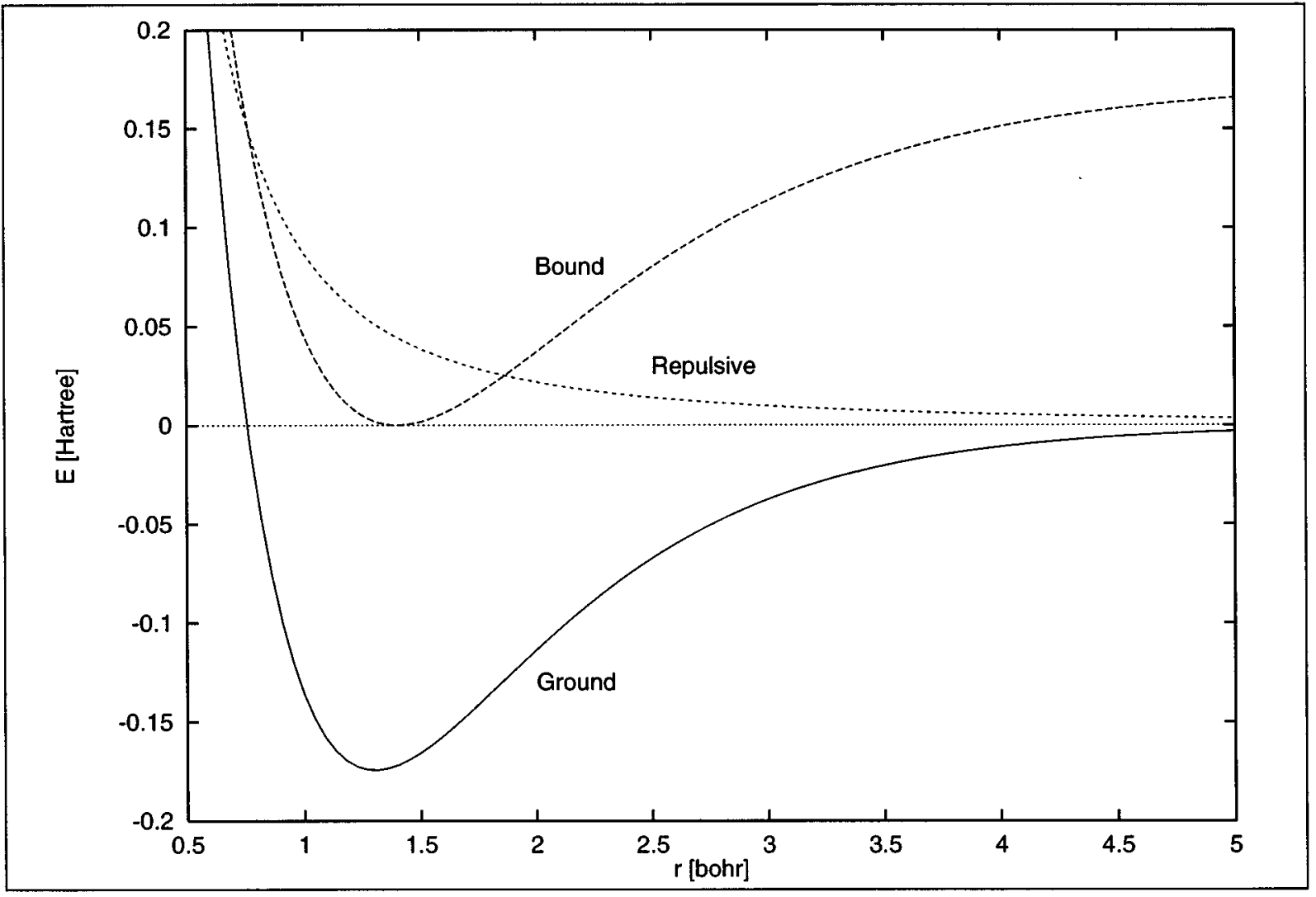

FIG. 1. A plot of the model diabatic potentials defined by Eq. (42), Eq. (43), and Eq. (44).

function, $C$ the coupling, and $R$ the field, which in the rotating wave approximation takes the form ${ }^{14}$

$$
\hat{R}_{b g}(t)=\hat{R}_{g b}^{\dagger}(t)=-\frac{1}{2} a(t) E_{0} e^{-i \omega t} \mu_{E},
$$

where $a(t)$ is the envelope function, $E_{0}$ the field amplitude, and $\mu_{E}$ the transition dipole moment in the direction of the field.

\section{A. The field as a perturbation}

We now approach the problem using time-dependent perturbation theory. We write the full Hamiltonian as $\hat{H}=\hat{H}_{0}+\hat{H}^{\prime}(t)$, and assume $\hat{H}^{\prime}(t)$ to be small enough to justify a perturbation treatment. Now let the unperturbed Hamiltonian be the full Hamiltonian, Eq. (1), without the field and hence let

$$
\hat{H}^{\prime}(t)=\left[\begin{array}{ccc}
0 & \hat{R}_{g b}(t) & 0 \\
\hat{R}_{b g}(t) & 0 & 0 \\
0 & 0 & 0
\end{array}\right] .
$$

Now assume that at $t=0$ we have prepared the system in an eigenstate of the electronic ground state Hamiltonian $\hat{H}_{g}$, hence we have

$$
\left[\begin{array}{c}
\left|\chi_{g}(0)\right\rangle \\
\left|\chi_{b}(0)\right\rangle \\
\left|\chi_{r}(0)\right\rangle
\end{array}\right]=\left[\begin{array}{c}
\left|n_{g}\right\rangle \\
0 \\
0
\end{array}\right]
$$

The unperturbed evolution operator, $\hat{U}_{0}(t)$ becomes

$$
\hat{U}_{0}(t)=\left[\begin{array}{cc}
e^{-i / \hbar \hat{H}_{g} t} & 0 \\
0 & e^{-i / \hbar}\left[\begin{array}{cc}
\hat{H}_{b} & \hat{C}_{b r} \\
\hat{C}_{r b} & \hat{H}_{r}
\end{array}\right]^{t}
\end{array}\right] .
$$

Recall that a function of an operator or a matrix is defined through its series, and we can hence easily evaluate $\hat{U}_{0}(t)$ using either the Chebychev or the SOD scheme. ${ }^{15}$ Using first order perturbation theory we can write an expression for the state of the nuclei in the two upper electronic states,

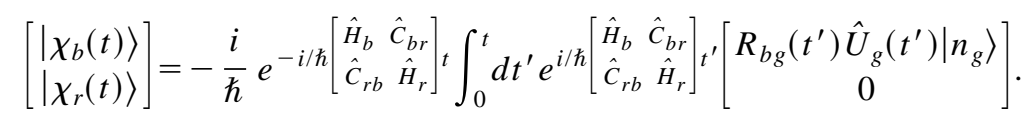


Further, if we define $E=E_{n}^{g}+\hbar \omega$, where $E_{n}^{g}$ is the energy of $\left|n_{g}\right\rangle$ we can write

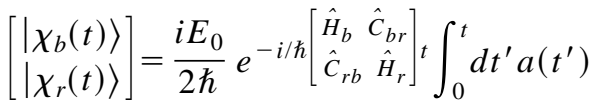

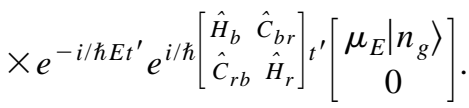

Delta-distribution limit for the light source, $a(t)=\delta(t)$, yields

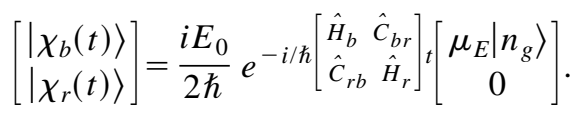

We see that we simply displace the initial state vertically up into the upper potentials and propagate it from here. We hence have a true Franck-Condon transition. The transition probability to the repulsive state can (above the tunneling regime) be related to the Landau-Zener formula. ${ }^{16}$

Now in the continuous wave limit we set

$$
a\left(t^{\prime}\right)=\left\{\begin{array}{l}
1 \text { for } 0 \leqslant t^{\prime} \leqslant \tau, \quad \tau \text { large } \\
0 \text { for } \tau<t^{\prime}
\end{array}\right.
$$

and calculate the transition probability to the repulsive potential by

$$
P_{r}(t)=\left\langle\chi_{r}(t) \mid \chi_{r}(t)\right\rangle
$$

which again can be evaluated as the absolute square of $\left|\chi_{r}(t)\right\rangle$ calculated by

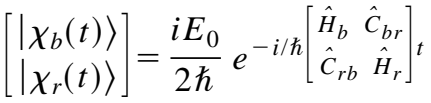

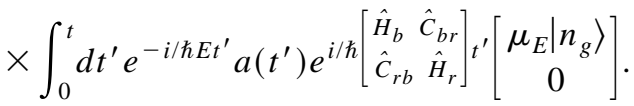

It should be emphasized that the only approximation in Eq. (11) is a perturbation treatment for the field, and this can always be accommodated by using a weak field. We will in the following use Eq. (11) as a reference that we can compare the more approximative formulas against.

\section{B. The field and the coupling as perturbations}

We shall now assume that the coupling also is small enough to justify a perturbation treatment. The transition from the ground state to the bound excited state, and further to the dissociative state is a second order process and we have to use second order perturbation theory. The perturbation Hamiltonian now becomes

$$
\hat{H}^{\prime}(t)=\left[\begin{array}{ccc}
0 & \hat{R}_{g b}(t) & 0 \\
\hat{R}_{b g}(t) & 0 & \hat{C}_{b r} \\
0 & \hat{C}_{r b} & 0
\end{array}\right] .
$$

And the unperturbed Hamiltonian hence becomes diagonal, and we get for the evolution operator $\hat{U}_{0}(t)$,

$$
\hat{U}_{0}(t)=\left[\begin{array}{ccc}
e^{-i / \hbar \hat{H}_{g} t} & 0 & 0 \\
0 & e^{-i / \hbar \hat{H}_{b} t} & 0 \\
0 & 0 & e^{-i / \hbar \hat{H}_{r} t}
\end{array}\right] .
$$

Now second order perturbation theory yields for $\left|\chi_{r}(t)\right\rangle$,

$$
\begin{aligned}
\left|\chi_{r}(t)\right\rangle= & \left(\frac{i}{\hbar}\right)^{2} \hat{U}_{r}(t) \int_{0}^{t} d t^{\prime} \int_{0}^{t^{\prime}} d t^{\prime \prime} \hat{U}_{r}^{\dagger}\left(t^{\prime}\right) \\
& \times \hat{C}_{r b} \hat{U}_{b}\left(t^{\prime}\right) \hat{U}_{b}^{\dagger}\left(t^{\prime \prime}\right) \hat{R}_{b g}\left(t^{\prime \prime}\right) \hat{U}_{g}\left(t^{\prime \prime}\right)\left|n_{g}\right\rangle .
\end{aligned}
$$

We consider the continuous wave limit, Eq. (9), and we get

$$
\begin{aligned}
\left|\chi_{r}(t)\right\rangle= & \frac{E_{0}}{2 \hbar^{2}} \hat{U}_{r}(t) \int_{0}^{t} d t^{\prime} \int_{0}^{t^{\prime}} d t^{\prime \prime} \hat{U}_{r}^{\dagger}\left(t^{\prime}\right) \hat{C}_{r b} \\
& \times \hat{U}_{b}\left(t^{\prime}\right) a\left(t^{\prime \prime}\right) e^{i / \hbar\left(\hat{H}_{b}-E\right) t^{\prime \prime}} \mu_{E}\left|n_{g}\right\rangle,
\end{aligned}
$$

where we again have defined $E=E_{n}^{g}+\hbar \omega$, and used that $\left|n_{g}\right\rangle$ is an eigenstate of the electronic ground state Hamiltonian $\hat{H}_{g}$.

Now consider the second of the integrals in Eq. (15), we shall denote this integral by the symbol $\left|\mathscr{B}_{b}\left(t^{\prime}\right)\right\rangle$ and name it a Raman wave function ${ }^{6}$ (see also Ref. 17). We implicitly assume that $t^{\prime}<\tau$. Note that we define the Raman wave function as a time dependent object, and not as in Ref. 6 take the $\infty$-limit. As we shall see taking the $\infty$-limit causes the bound state Raman wave function to diverge!

Consider

$$
\left|\mathscr{R}_{b}\left(t^{\prime}\right)\right\rangle=\int_{0}^{t^{\prime}} d t^{\prime \prime} e^{i / \hbar\left(\hat{H}_{b}-E\right) t^{\prime \prime}}|\varphi\rangle
$$

with $|\varphi\rangle=\mu_{E}\left|n_{g}\right\rangle$. Now insert a closure in the eigenstates of $\hat{H}_{b}$, and get

$$
\left|\mathscr{R}_{b}\left(t^{\prime}\right)\right\rangle=\sum_{m} \int_{0}^{t^{\prime}} d t^{\prime \prime} e^{i / \hbar\left(E_{m}^{b}-E\right) t^{\prime \prime}}\left|m_{b}\right\rangle\left\langle m_{b} \mid \varphi\right\rangle .
$$

We assume that we have tuned the frequency so that $E=E_{n}^{b}$, and hence we get for the $m_{b}=n_{b}$ term in the sum

$$
\int_{0}^{t^{\prime}} d t^{\prime \prime}\left|n_{b}\right\rangle\left\langle n_{b} \mid \varphi\right\rangle=t^{\prime}\left\langle n_{b} \mid \varphi\right\rangle\left|n_{b}\right\rangle .
$$

The other terms in the sum can be evaluated to

$$
\sum_{m \neq n}-i \hbar \frac{e^{i / \hbar\left(E_{m}^{b}-E\right) t^{\prime}}-1}{E_{m}^{b}-E}\left\langle m_{b} \mid \varphi\right\rangle\left|m_{b}\right\rangle .
$$

We see that for long times $t^{\prime} \sim \infty$, the $m_{b}=n_{b}$ term will dominate. We hence write for the Raman wave function,

$$
\left|\mathscr{R}_{b}\left(t^{\prime}\right)\right\rangle \underset{t^{\prime} \sim \infty}{\simeq} t^{\prime}\left\langle n_{b} \mid \varphi\right\rangle\left|n_{b}\right\rangle .
$$

Using this result we are now able to reduce Eq. (15) further. We shall consider two cases; first what happens while the field is on, $t<\tau$, and secondly what happens long time after the field is removed again, $t \gg \tau$.

First for $t<\tau$, we get 


$$
\begin{aligned}
\left|\chi_{r}(t)\right\rangle & =\frac{E_{0}}{2 \hbar^{2}} \hat{U}_{r}(t) \int_{0}^{t} d t^{\prime} \hat{U}_{r}^{\dagger}\left(t^{\prime}\right) \hat{C}_{r b} \hat{U}_{b}\left(t^{\prime}\right)\left|\mathscr{B}_{b}\left(t^{\prime}\right)\right\rangle \\
& \simeq \frac{E_{0}}{2 \hbar^{2}}\left\langle n_{b} \mid \varphi\right\rangle \hat{U}_{r}(t) \int_{0}^{t} d t^{\prime} t^{\prime} \hat{U}_{r}^{\dagger}\left(t^{\prime}\right) \hat{C}_{r b} \hat{U}_{b}\left(t^{\prime}\right)\left|n_{b}\right\rangle,
\end{aligned}
$$

where we have assumed that the Raman wave function becomes proportional to an eigenstate immediately-this is not the case, but for long times, $t \sim \infty$ the error introduced by this assumption will vanish. We now use that $\left|n_{b}\right\rangle$ is an eigenstate of $\hat{H}_{b}$ and write

$$
\left|\chi_{r}(t)\right\rangle=\frac{E_{0}}{2 \hbar^{2}}\left\langle n_{b} \mid \varphi\right\rangle \hat{U}_{r}(t) \int_{0}^{t} d t^{\prime} t^{\prime} e^{i / \hbar\left(\hat{H}_{r}-E_{n}^{b}\right) t^{\prime}} \hat{C}_{r b}\left|n_{b}\right\rangle .
$$

From this equation we can easily evaluate the transition probability by forming the absolute square, we get

$\left.P_{r}(t)=\frac{E_{0}^{2}}{4 \hbar^{4}}\left|\left\langle n_{b}\left|\mu_{E}\right| n_{g}\right\rangle\right|^{2}\left|\int_{0}^{t} d t^{\prime} t^{\prime} e^{i / \hbar\left(\hat{H}_{r}-E_{n}^{b}\right) t^{\prime}} \hat{C}_{r b}\right| n_{b}\right\rangle\left.\right|^{2}$.

We see that we have now written the transition probability as the product of a Franck-Condon factor and a time dependent integral, though this is a great simplification compared to Eq. (11), we can in fact simplify Eq. (23) further. We insert a closure in the eigenstates of $\hat{H}_{r}$, and consider the integral part of Eq. (23) for $t \sim \infty$, in general we can write it as

$$
f_{t}(x)=\int_{0}^{t} d t^{\prime} \int_{0}^{t} d t^{\prime \prime} t^{\prime} e^{i x t^{\prime}} t^{\prime \prime} e^{-i x t^{\prime \prime}}
$$

We shall now rewrite this integral into a delta-distribution. First we normalize with respect to $x$,

$$
\int_{-\infty}^{\infty} d x f_{t}(x)=\int_{-\infty}^{\infty} d x \int_{0}^{t} d t^{\prime} \int_{0}^{t} d t^{\prime \prime} t^{\prime} t^{\prime \prime} e^{i x\left(t^{\prime}-t^{\prime \prime}\right)},
$$

now use that evaluating the integral over $x$ yields a deltadistribution,

$$
\begin{aligned}
\int_{-\infty}^{\infty} d x f_{t}(x) & =2 \pi \int_{0}^{t} d t^{\prime} \int_{0}^{t} d t^{\prime \prime} t^{\prime} t^{\prime \prime} \delta\left(t^{\prime}-t^{\prime \prime}\right) \\
& =2 \pi \int_{0}^{t} d t^{\prime} t^{\prime 2}=\frac{2 \pi}{3} t^{3} .
\end{aligned}
$$

Evaluating the integrals over $t^{\prime}$ and $t^{\prime \prime}$ in Eq. (24), yields

$$
t^{-3} f_{t}(x)=2 \frac{1-\cos (x t)}{x^{4} t^{3}}-2 \frac{\sin (x t)}{x^{3} t^{2}}+\frac{1}{x^{2} t} .
$$

We now easily see that for $x \neq 0$ we have that $\lim _{t \rightarrow \infty} t^{-3} f_{t}(x)=0$ and hence we can write

$$
\lim _{t \rightarrow \infty} t^{-3} f_{t}(x)=\frac{2 \pi}{3} \delta(x)
$$

hence for large $t$ we can reduce Eq. (23) to

$$
P_{r}(t)=\frac{\pi E_{0}^{2}}{6 \hbar^{3}}\left|\left\langle n_{b}\left|\mu_{E}\right| n_{g}\right\rangle\right|^{2}\left|\left\langle\epsilon_{r}=E_{n}^{b}\left|\hat{C}_{r b}\right| n_{b}\right\rangle\right|^{2} t^{3},
$$

where we have performed the integration over the eigenstates of $\hat{H}_{r}$, leaving only $\epsilon_{r}=E_{n}^{b}$.

We shall now consider the case with $t \gg \tau$. Here we can ignore the creation process while the field was on, and we write Eq. (22) as

$$
\left|\chi_{r}(t)\right\rangle=\frac{\tau E_{0}}{2 \hbar^{2}}\left\langle n_{b}\left|\mu_{E}\right| n_{g}\right\rangle \hat{U}_{r}(t) \int_{0}^{t} d t^{\prime} e^{i / \hbar\left(\hat{H}_{r}-E_{n}^{b}\right) t^{\prime}} \hat{C}_{r b}\left|n_{b}\right\rangle .
$$

Compare this with the definition of the bound state Raman wave function, Eq. (16). It is hence obvious to define a repulsive state Raman wave function by

$$
\left|\mathscr{R}_{r}(t)\right\rangle=\int_{0}^{t} d t^{\prime} e^{i / \hbar\left(\hat{H}_{r}-E\right) t^{\prime}}|\phi\rangle
$$

with $|\phi\rangle=\hat{C}_{r b}\left|n_{b}\right\rangle$, and with $E=E_{n}^{b}$ in the present case.

We shall now consider closer the properties of this repulsive state Raman wave function. First consider the norm of the Raman wave function

$$
\begin{aligned}
& \left\langle\mathscr{B}_{r}(t) \mid \mathscr{B}_{r}(t)\right\rangle \\
& \quad=\int_{0}^{t} d t^{\prime} \int_{0}^{t} d t^{\prime \prime}\left\langle\phi\left|e^{-i / \hbar\left(\hat{H}_{r}-E\right) t^{\prime}} e^{i / \hbar\left(\hat{H}_{r}-E\right) t^{\prime \prime}}\right| \phi\right\rangle .
\end{aligned}
$$

Now let $\left|\epsilon_{r}\right\rangle$ be the eigenstate of $\hat{H}_{r}$ with energy $E$, and insert a closure of all eigenstates in Eq. (32), we get

$$
\begin{aligned}
& \left\langle\mathscr{B}_{r}(t) \mid \mathscr{B}_{r}(t)\right\rangle \\
& \quad=\int d \epsilon^{\prime}\left|\left\langle\epsilon^{\prime} \mid \phi\right\rangle\right|^{2} \int_{0}^{t} d t^{\prime} \int_{0}^{t} d t^{\prime \prime} e^{i / \hbar\left(\epsilon^{\prime}-E\right)\left(t^{\prime \prime}-t^{\prime}\right)} .
\end{aligned}
$$

Evaluating the integrals over $t^{\prime}$ and $t^{\prime \prime}$ yields

$$
\begin{aligned}
& \left\langle\mathscr{B}_{r}(t) \mid \mathscr{B}_{r}(t)\right\rangle \\
& \quad=2 \hbar^{2} \int d \epsilon^{\prime}\left|\left\langle\epsilon^{\prime} \mid \phi\right\rangle\right|^{2} \frac{1-\cos \left[\left(\epsilon^{\prime}-E\right) t / \hbar\right]}{\left(\epsilon^{\prime}-E\right)^{2}} .
\end{aligned}
$$

From Ref. 18 (p. 469) we find that

$$
\delta(x)=\lim _{t \rightarrow \infty} \frac{1-\cos (t x)}{\pi t x^{2}}
$$

and hence we can write for large $t$,

$$
\left\langle\mathscr{B}_{r}(t) \mid \mathscr{R}_{r}(t)\right\rangle_{t \sim \infty}^{\simeq} 2 \pi \hbar \int d \epsilon^{\prime} t\left|\left\langle\epsilon^{\prime} \mid \phi\right\rangle\right|^{2} \delta\left(\epsilon^{\prime}-E\right)
$$

evaluating the integral over $\epsilon^{\prime}$ yields

$$
\left\langle\mathscr{B}_{r}(t) \mid \mathscr{B}_{r}(t)\right\rangle_{t \sim \infty}^{\simeq} 2 \pi \hbar t\left|\left\langle\epsilon_{r}=E \mid \phi\right\rangle\right|^{2} .
$$

We now return to the definition of the Raman wave function Eq. (31), and formally evaluate the integral, we get,

$$
\left|\mathscr{R}_{r}(t)\right\rangle=-\frac{i \hbar\left[e^{i / \hbar\left(\hat{H}_{r}-E\right) t}-1\right]}{\hat{H}_{r}-E}|\phi\rangle .
$$

Multiplying by $\left(\hat{H}_{r}-E\right)$ now gives 


$$
\left(\hat{H}_{r}-E\right)\left|\mathscr{R}_{r}(t)\right\rangle=-i \hbar\left[e^{i / \hbar\left(\hat{H}_{r}-E\right) t}-1\right]|\phi\rangle .
$$

Now, since the norm of the Raman wave function is proportional to $t$, and since the norm of $|\phi\rangle$ is finite and independent of $t$, the only way Eq. (39) can be valid, is if for large $t$ the Raman wave function approaches an eigenstate of $\hat{H}_{r}$, namely the eigenstate with energy $E$.

It has earlier been shown ${ }^{6}$ that the real-part of the Raman wave function in the position representation will indeed approach an eigenstate of $\hat{H}_{r}$ for large $t$. The proof given above is much stronger, though.

Using these properties of the repulsive state Raman wave function together with Eq. (30) yields for the transition probability

$$
P_{r}(t)=\frac{\pi \tau^{2} E_{0}^{2}}{2 \hbar^{3}}\left|\left\langle n_{b}\left|\mu_{E}\right| n_{g}\right\rangle\right|^{2}\left|\left\langle\epsilon_{r}=E_{n}^{b}\left|\hat{C}_{r b}\right| n_{b}\right\rangle\right|^{2} t
$$

i.e., the well-known linear $t$ dependence.

We have now accomplished to make expressions of the transition probability simply as a product of Franck-Condon factors. In some cases, if the analytical expressions for the eigenfunctions exist we can evaluate Eq. (29) and Eq. (40) directly, however, in many cases it becomes simpler to evaluate $\left|\left\langle\epsilon_{r}\left|\hat{C}_{r b}\right| n_{b}\right\rangle\right|^{2}$ using $^{8}$

$2 \pi \hbar\left|\left\langle\epsilon_{r}=E_{n}^{b}\left|\hat{C}_{r b}\right| n_{b}\right\rangle\right|^{2}=\int_{-\infty}^{\infty} d t e^{i / \hbar E_{n}^{b} t}\left\langle\phi\left|e^{-i / \hbar \hat{H}_{r} t}\right| \phi\right\rangle$

with $|\phi\rangle=\hat{C}_{r b}\left|n_{b}\right\rangle$. We can think of the evaluation of the integral as composed by a vertical displacement (a true Franck-Condon transition) of a wave packet given as a product between a matrix element of the coupling and the prepared vibrational eigenstate, followed by a propagation in the repulsive electronic state. Here if the potential is strongly repulsive we need only to integrate up to short times, since the wave packet will then move very fast away from its initial position, and the self-overlap will vanish. The evaluation of Eq. (41) can be carried out exactly or even-often successfully—by approximate methods. ${ }^{19,8}$ The use of Eq. (41) is also from a conceptual point of view advantageous.

\section{RESULTS AND DISCUSSION}

In order to test the derived formulas we have made numerical calculations on a model example using the fast Fourier method in conjunction with the SOD time propagation scheme. ${ }^{15}$ We aim our interest on Eqs. (11), (23), and (29) since it is in between these formulas new approximation are made. In our model we consider a diatomic molecule, with the diabatic potentials,

$$
\begin{aligned}
& V_{g}(r)=D\left[e^{-\beta_{g}\left(r-r_{g}\right)}-1\right]^{2}-D, \\
& V_{b}(r)=D\left[e^{-\beta_{b}\left(r-r_{b}\right)}-1\right]^{2}, \\
& V_{r}(r)=\frac{1}{2} D / r^{2},
\end{aligned}
$$

with the parameters $D=0.1744, \beta_{\mathrm{g}}=1.277, r_{g}=1.3020$, $\beta_{b}=1.0277$, and $r_{b}=1.4020$ (all in atomic units). A plot of the potentials is shown in Fig. 1. For the coupling we use a Gaussian

$$
C_{b r}(r)=C_{r b}(r)=A e^{-b^{2}\left(r-r_{i}\right)^{2}},
$$

where $A=10^{-4}, b=2$ and $r_{i}$, the intersection between $V_{b}$ and $V_{r}$, equals 1.8656. Further we set $\mu_{E}=10^{-4}$. The reduced mass of the system is $m=918.075$. We measure the transition probability in units of $E_{0}^{2}$ and shall hence only assume that it is chosen within the perturbation limit. With these parameters we get for the bound excited state a vibration period of 7.6 fs (compare with Fig. 5).

We have calculated the dissociation probabilities $P_{r}^{00}$, $P_{r}^{01}$, and $P_{r}^{02}$ representing the transition from the 0th eigenstate in the electronic ground state via the 0 th, 1 st, and 2 nd eigenstate in the bound electronic excited state. Plots of the dissociation probabilities are shown in Figs. 2, 3, and 4, respectively.

First consider Fig. 2, here we see a very fine agreement between Eq. (11) and Eq. (23), but a very bad agreement with Eq. (29). Next consider Fig. 3, here we see indeed very good agreement between all the three formulas, and we shall hence conclude that here all the approximations are valid. Finally, consider Fig. 4 here we see that the approximations leading us from Eq. (11) to Eq. (23) are not valid for short times, however, for long times the relative error decreases, and the approximations becomes better. Further we see that we practically do not introduce any further errors in using Eq. (29).

\section{A. Validity of approximations}

Based on the calculations, we shall now reconsider the approximations one by one. First of all we demand that the nonadiabatic terms in the Hamiltonian are small enough in order to justify a perturbation treatment. Or more formally,

$$
\frac{t\left\|\hat{H}^{\prime}\right\|}{\hbar} \ll 1 .
$$

Violating this inequality will cause the probabilities calculated by Eq. (11) and Eq. (23) to diverge for long times. In our calculations we are not met with this problem, and hence we shall not treat it any further.

The next approximation made, is that we assume that an eigenstate is created immediately in the bound electronic excited state. Consider again the formation of the Raman wave function Eq. (17), and write the sum as

$$
\begin{aligned}
\left|\mathscr{R}_{b}\left(t^{\prime}\right)\right\rangle= & t^{\prime}\left\langle n_{b} \mid \varphi\right\rangle\left|n_{b}\right\rangle \\
& -i \hbar \sum_{m \neq n} \frac{e^{i / \hbar\left(E_{m}^{b}-E_{n}^{b}\right) t^{\prime}}-1}{E_{m}^{b}-E_{n}^{b}}\left\langle m_{b} \mid \varphi\right\rangle\left|m_{b}\right\rangle .
\end{aligned}
$$

We seek the conditions for which the first term will dominate, and hence we shall establish an upper bound for the second term 


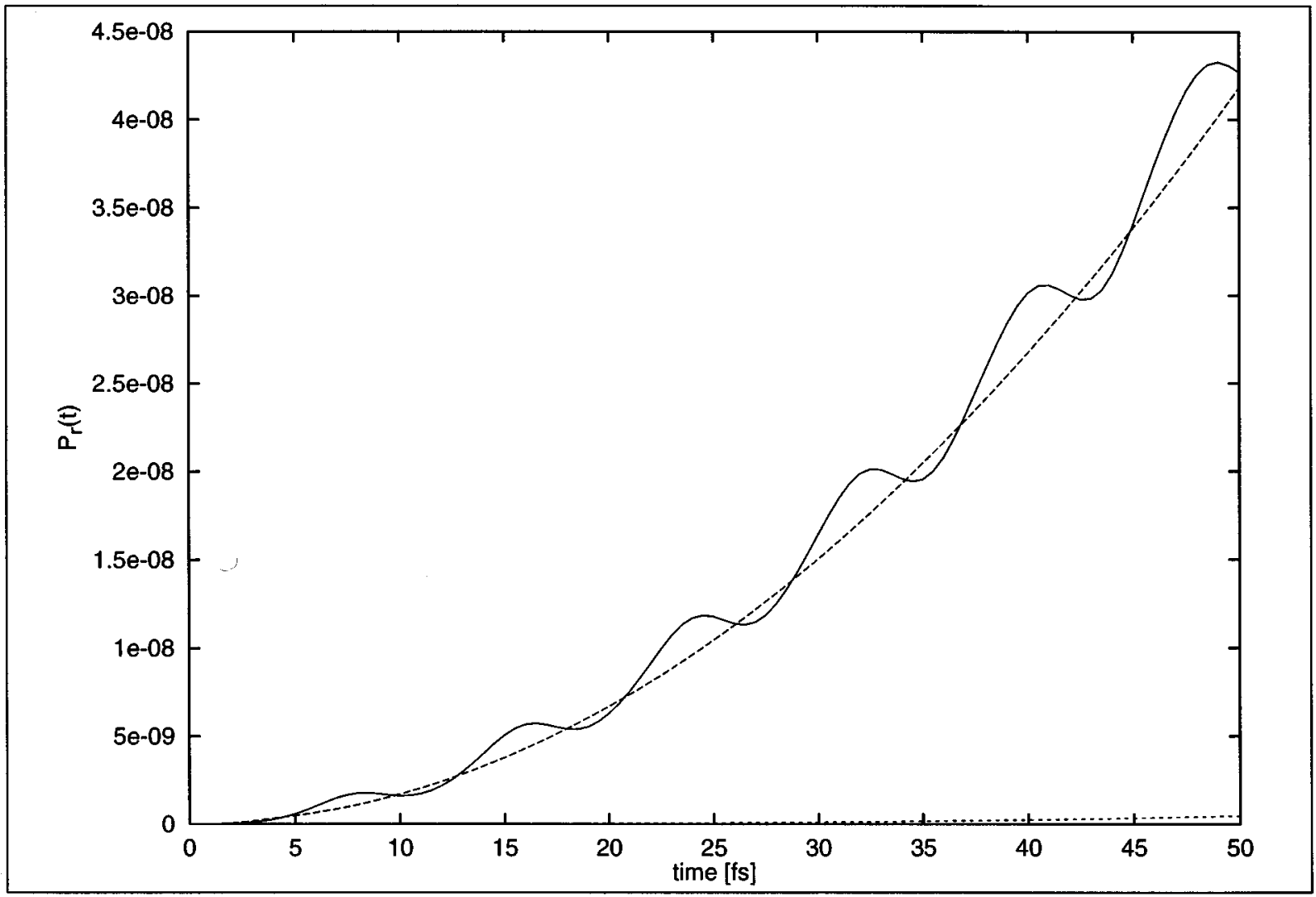

FIG. 2. The 00-transition probability calculated using Eqs. (11), (23), and (29) plotted in solid, long-dashed, and short-dashed lines, respectively.

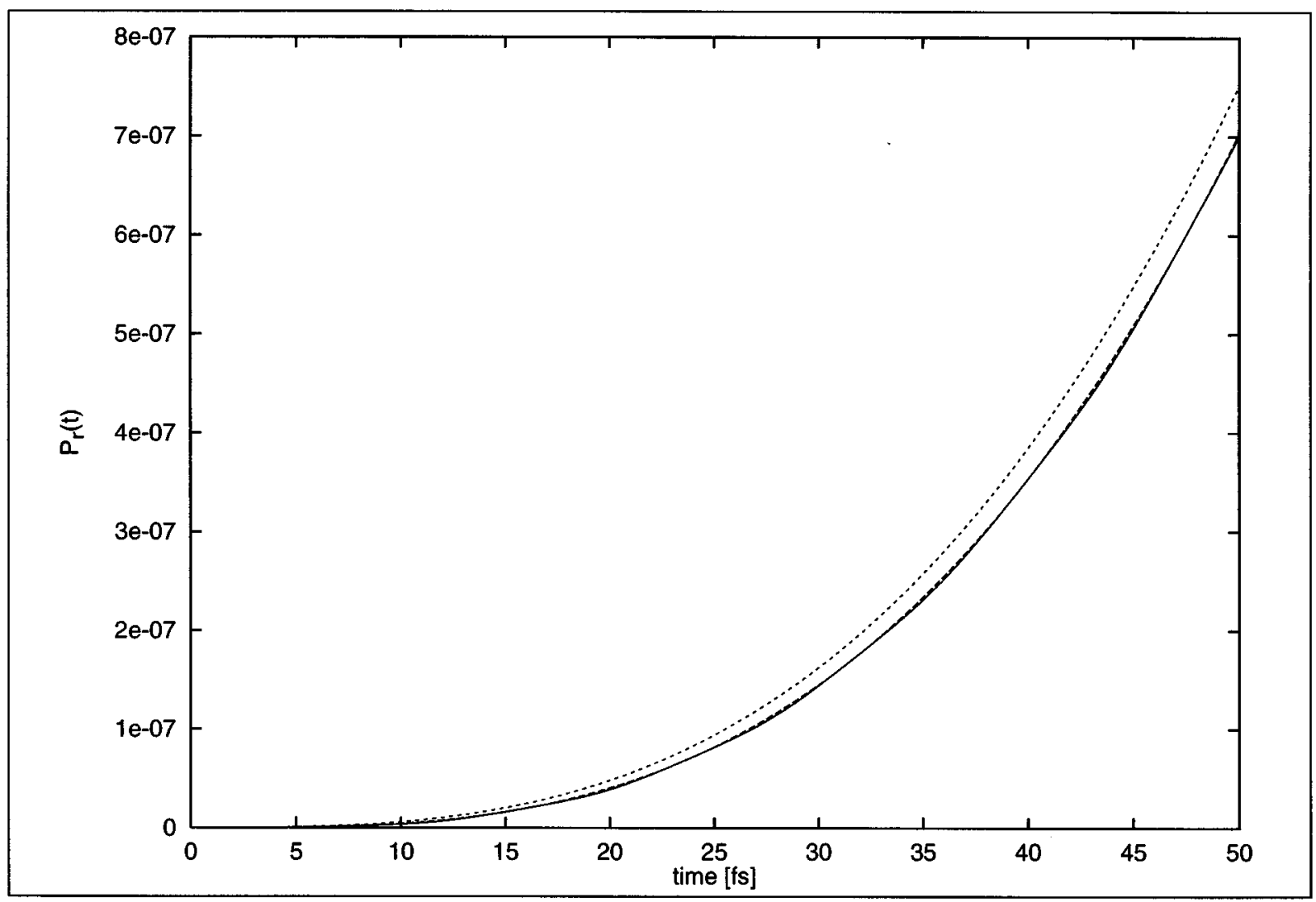

FIG. 3. The 01-transition probability calculated using Eqs. (11), (23), and (29) plotted in solid, long-dashed, and short-dashed lines, respectively.

J. Chem. Phys., Vol. 104, No. 9, 1 March 1996 


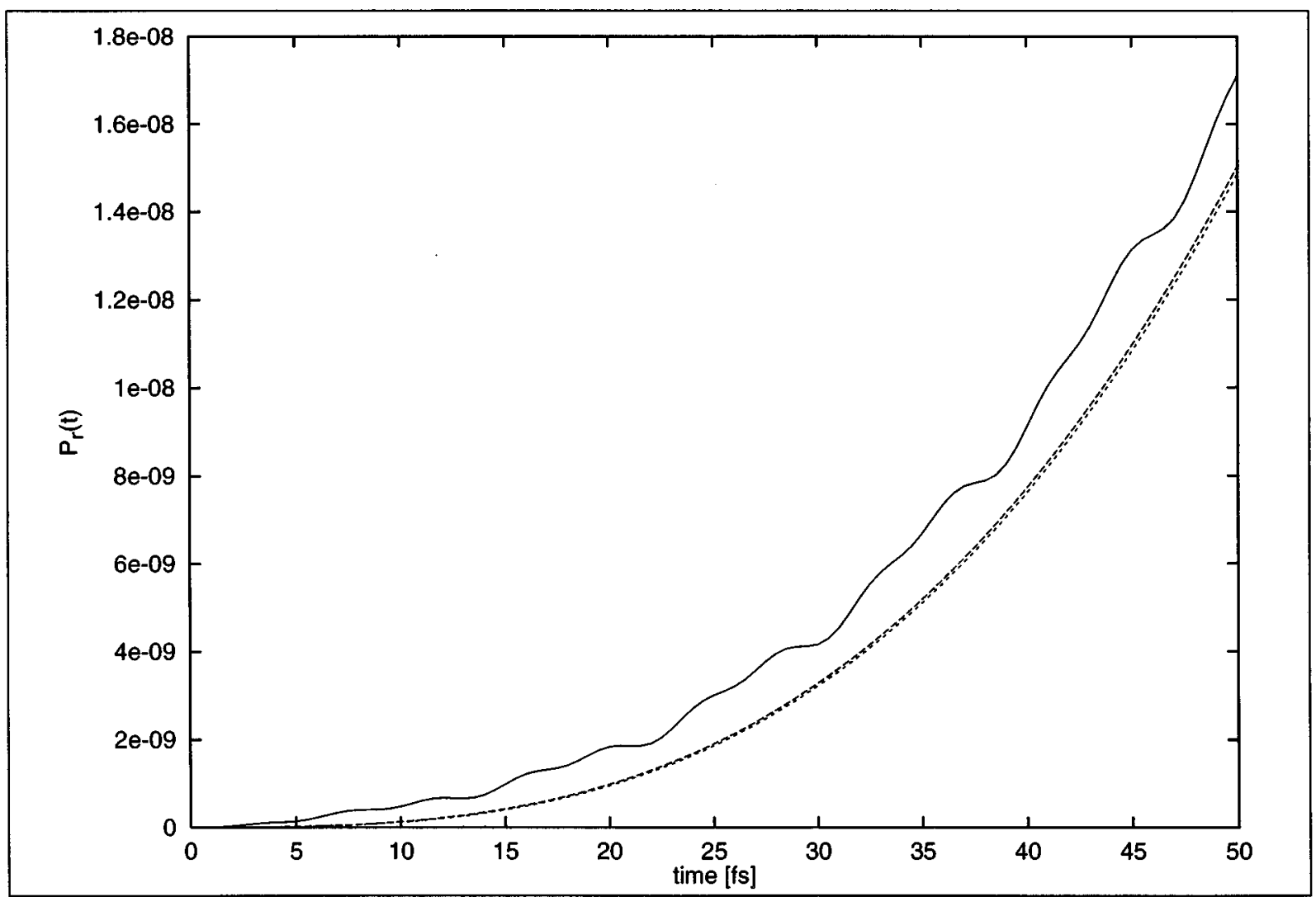

FIG. 4. The 02-transition probability calculated using Eqs. (11), (23), and (29) plotted in solid, long-dashed, and short-dashed lines, respectively.

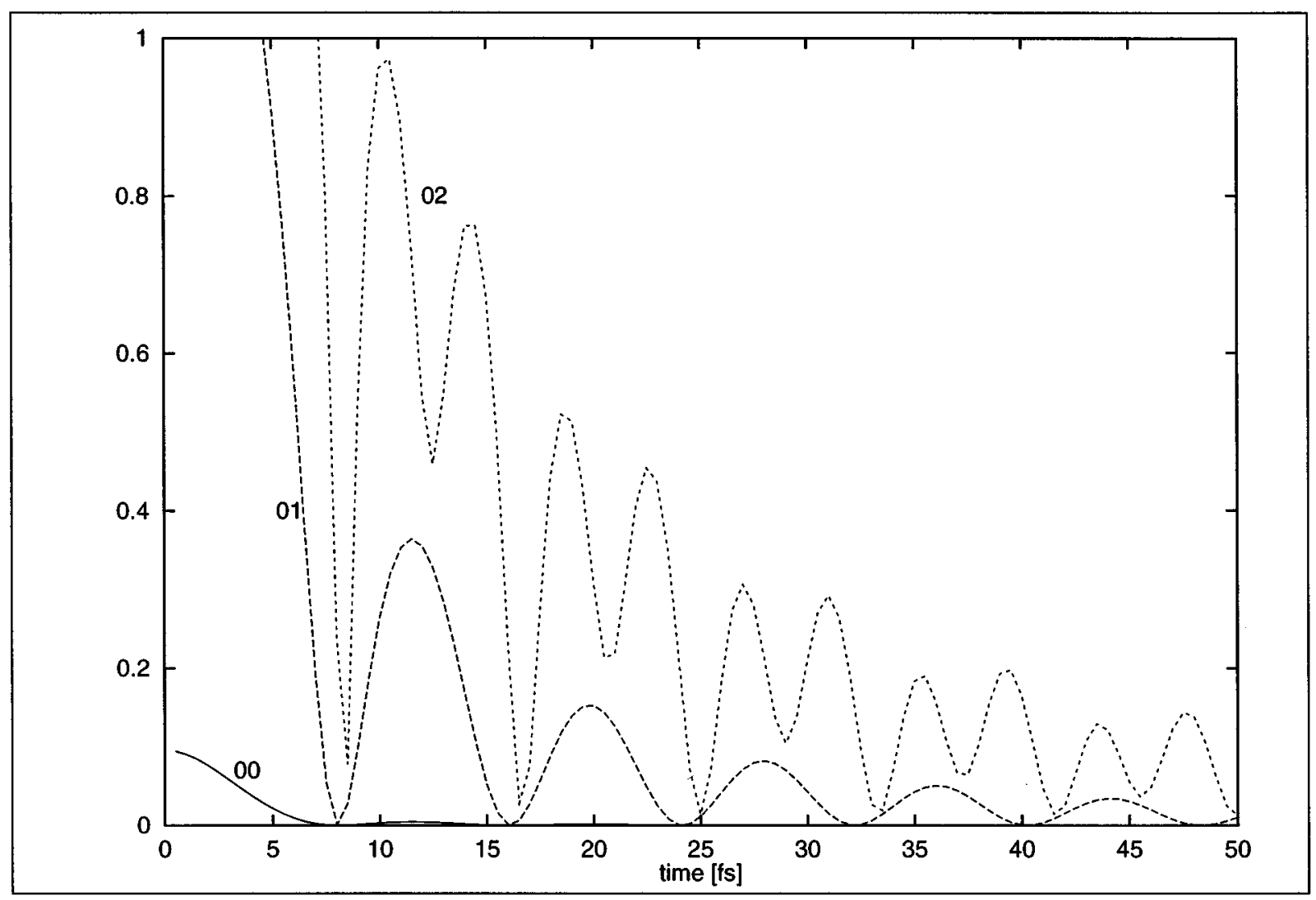

FIG. 5. The formation of an eigenstate, according to Eq. (51), in the bound electronic excited state.

J. Chem. Phys., Vol. 104, No. 9, 1 March 1996 


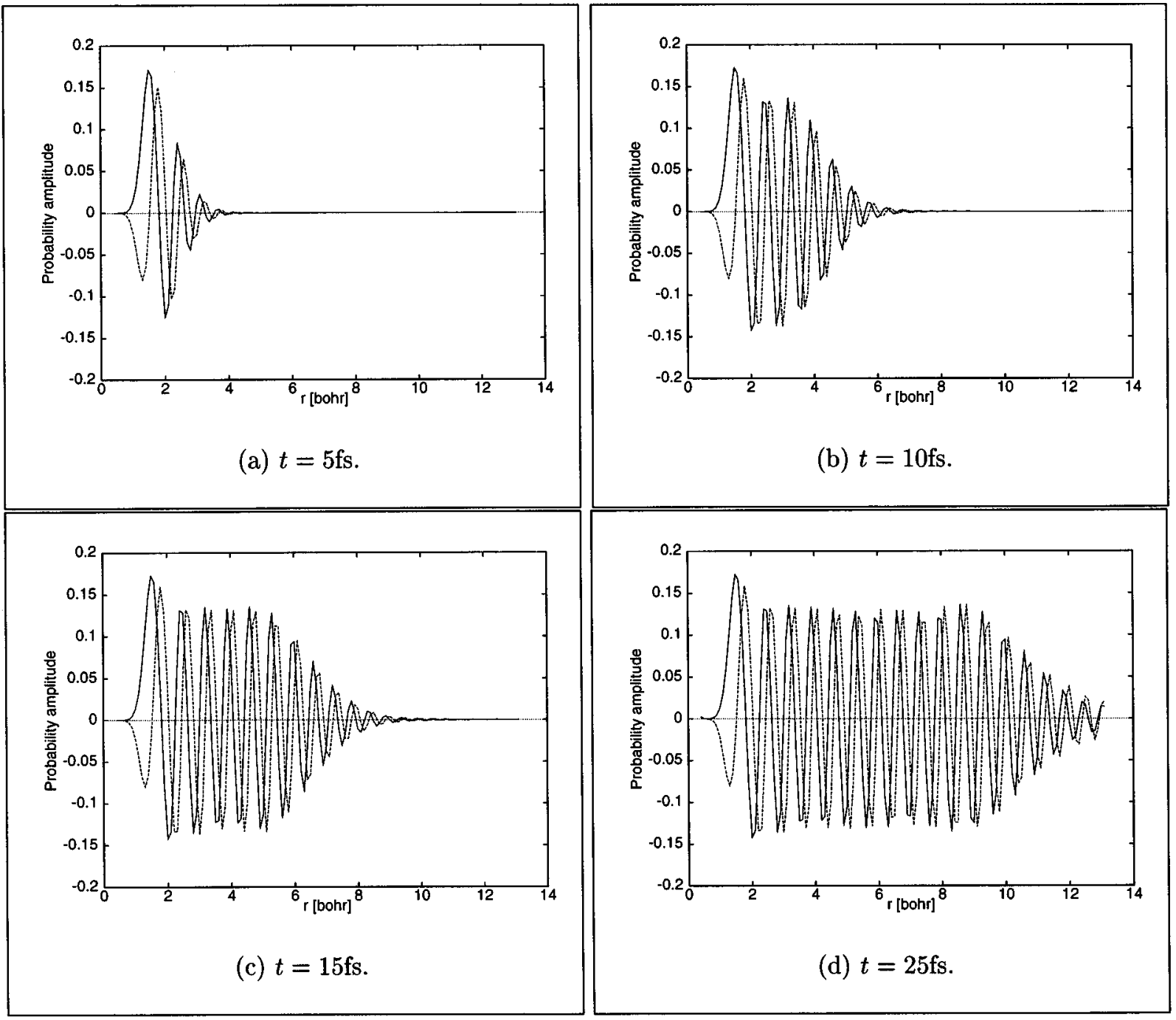

FIG. 6. The Raman wave function created in the repulsive potential for the 01-transition. The solid curve is the real-part and the dashed curve the imaginary-part of the Raman wave function calculated via the FFT-method.

$$
\begin{gathered}
\|-i \hbar \sum_{m \neq n} \frac{e^{i / \hbar\left(E_{m}^{b}-E_{n}^{b}\right) t^{\prime}}-1}{E_{m}^{b}-E_{n}^{b}}\left\langle m_{b} \mid \varphi\right\rangle\left|m_{b}\right\rangle \| \\
\leqslant 2 \hbar \max _{m \neq n} \frac{1}{E_{m}^{b}-E_{n}^{b}} \| \sum_{m \neq n}\left\langle m_{b} \mid \varphi\right\rangle\left|m_{b}\right\rangle \| \\
\leqslant 2 \hbar \sqrt{\|\varphi\|^{2}-\left|\left\langle n_{b} \mid \varphi\right\rangle\right|^{2}} \max _{m \neq n} \frac{1}{E_{m}^{b}-E_{n}^{b}}
\end{gathered}
$$

further the norm of the first term evaluates to $t^{\prime}\left|\left\langle n_{b} \mid \varphi\right\rangle\right|$, and we get the inequality

$$
t^{\prime}\left|\left\langle n_{b} \mid \varphi\right\rangle\right| \gg 2 \hbar \sqrt{\|\varphi\|^{2}-\left|\left\langle n_{b} \mid \varphi\right\rangle\right|^{2}} \max _{m \neq n} \frac{1}{E_{m}^{b}-E_{n}^{b}}
$$

or

$$
t^{\prime} \gg 2 \hbar \frac{\sqrt{\|\varphi\|^{2}-\left|\left\langle n_{b} \mid \varphi\right\rangle\right|^{2}}}{\left|\left\langle n_{b} \mid \varphi\right\rangle\right|} \max _{m \neq n} \frac{1}{E_{m}^{b}-E_{n}^{b}}
$$

We hence see that the overlap between $|\varphi\rangle$ and the eigenstate in the bound electronic excited state $\left|n_{b}\right\rangle$, is very essential to how fast the Raman wave function becomes proportional to $\left|n_{b}\right\rangle$. We will hence expect that we for the 00-transition succeeds to create an eigenstate much faster than for the $02-$ transition. In Fig. 5 we plot the quantity

$$
\| \frac{\left|\mathscr{R}_{b}(t)\right\rangle}{\left\|\mathscr{R}_{b}(t)\right\|}-\left|n_{b}\right\rangle \|
$$

to monitor how fast the Raman wave function becomes proportional to $\left|n_{b}\right\rangle$. We see that the Raman wave function indeed becomes proportional to $\left|n_{b}\right\rangle$ much faster for the 00transition than for the 01- and 02-transition. The fact that it takes quite some time before the Raman wave function becomes proportional to $\left|n_{b}\right\rangle$ for the 02-transition is also reflected in Fig. 4 where the agreement between Eq. (11) and Eq. (23) is not good for short times.

We shall now consider the approximations introduced from Eq. (23) to Eq. (29), i.e., in the formation of the delta- 


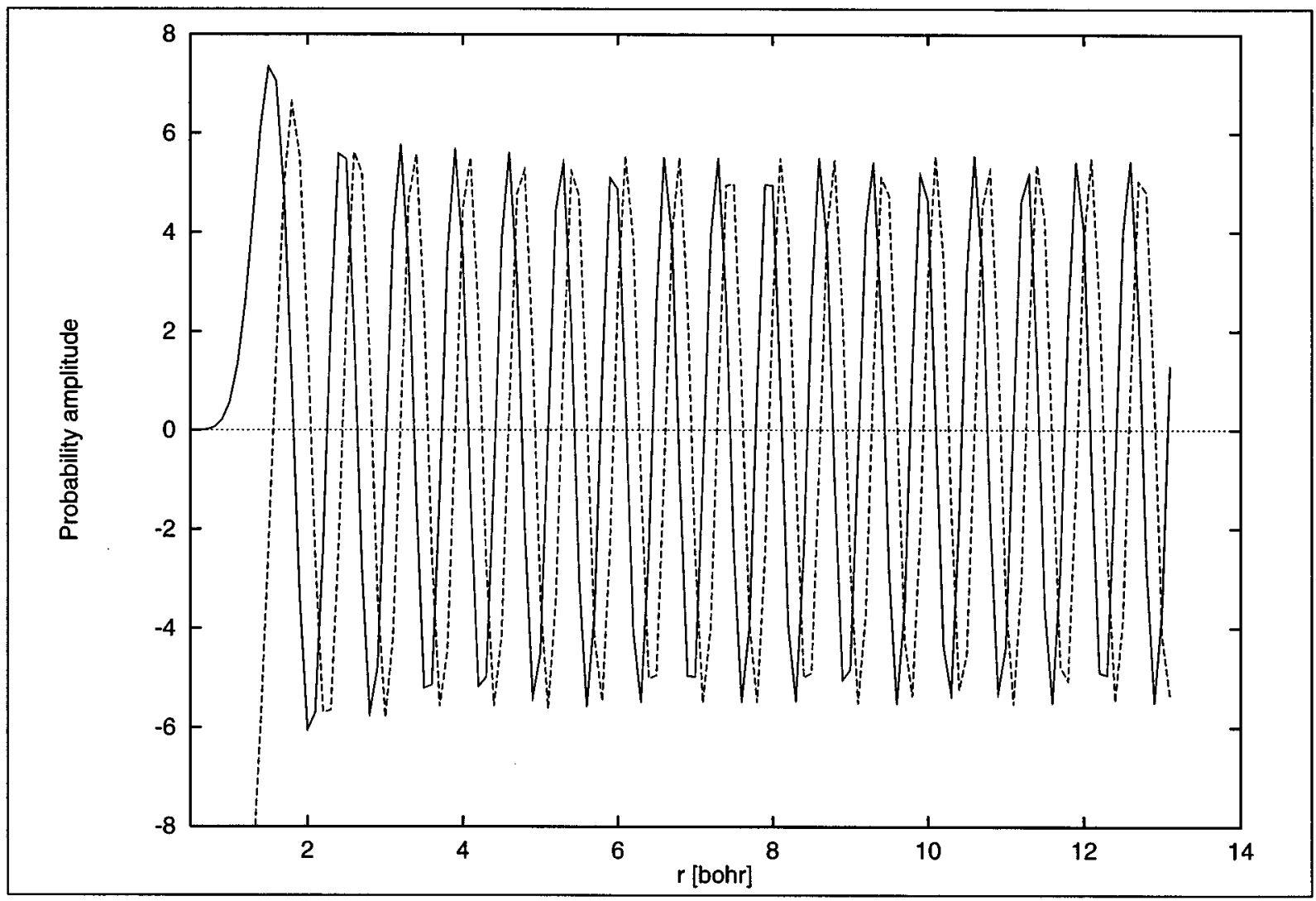

FIG. 7. The outgoing eigenfunction for a $1 / r^{2}$-potential calculated analytically using the same parameters as in the calculation of the Raman wave function on the repulsive potential. The solid line is the real-part, the dashed the imaginary-part.

distribution. The problem here is analogous to the one just described above; if the quantity $\left\langle\epsilon_{r}=E_{n}^{b}\left|\hat{C}_{r b}\right| n_{b}\right\rangle$ is very small compared to its value at other values of $\epsilon_{r}$, then it will take quite some time before the delta-distribution is peeked enough to filter off these other contributions. This is what causes Eq. (29) to fail in Fig. 2. However, for very long times it will give the right transition probability.

Finally, we shall make a note on how the repulsive state Raman wave function looks. We know that it is an eigenfunction of the Hamiltonian $\hat{H}_{r}$. We get an eigenstate that do not satisfy the usual boundary condition; to vanish at the origin. This eigenfunction is in fact a Riccati-Hankel function. ${ }^{20}$ Figure 6 shows how the Raman wave function is created in time, and Fig. 7 shows the outgoing eigenstate for the $r^{-2}$ potential (a Ricciati-Hankel function). We see that as $t \rightarrow \infty$, the Raman wave function becomes proportional to the outgoing eigenstate.

\section{B. Franck-Condon principle for electronic predissociation}

The Franck-Condon principle was originally formulated for electronic transitions due to light absorption. ${ }^{21,22}$ It is well-known that the impulsive excitation induced by a very short light pulse gives the quantum mechanical counterpart of the (semiclassical) proposition formulated by Franck and Condon. ${ }^{9,2}$

The wave packet created in such an instantaneous transition can, however, also play a central role in the expression for the absorption probability when the molecule is irradiated by a time-dependent continuous wave field. ${ }^{8,9}$ For electronic predissociation the action of the time-independent perturbation is very similar, the only difference being that this constant perturbation only allows for transitions between states with the same energy and an expression, Eq. (41), which contains an instantaneous nuclear transition can again be formulated. It can be very useful, conceptually and numerically, to use this formulation which is suggestive of the FranckCondon principle. However, the real process involves, as demonstrated above, a continuous transition.

\section{CONCLUSIONS}

The transition probabilities for radiationless transitions are often expressed in terms of Franck-Condon factors. We have considered the validity of this approach. First, a vibrational eigenstate should be created instantaneously. This requirement is, in general, not fulfilled in practice and it might take of the order of several vibrational periods before an 
eigenstate is created (we have in this paper explicitly considered a one-dimensional system, for a large molecule a typical vibrational period might not give the right estimate of the required time if the intramolecular vibrational energy transfer is slow in the bound part of the potential). Second, the time should be sufficiently large to ensure energy conservation in the radiationless transition and at the same time not larger than the limit permitted by perturbation theory. A small value of the coupling $\hat{C}_{r b}$ together with large values of the overlaps $\left\langle n_{b}\left|\mu_{E}\right| n_{g}\right\rangle$ and $\left\langle\epsilon_{r}\left|\hat{C}_{r b}\right| n_{b}\right\rangle$ (compared to overlaps at different values than $n_{b}$ and $\epsilon_{r}$ ) will reduce the restrictions imposed by both of the above listed requirements and ensure that the description can give a fair estimate of the transition probability.

We analyzed the nature of the Raman wave function for bound and repulsive states (one step further than in Ref. 6). The Raman wave function created in a bound potential is, at sufficiently large times, an eigenstate which grows linearly in time. For a real-valued initial state the Raman wave function is also real-valued. The Raman wave function created in a repulsive potential is, at sufficiently large times, an eigenstate which asymptotically becomes an outgoing plane wave. Thus this function carries both a real and an imaginary part.

We pointed out that transformation of the FranckCondon factors for the radiationless transition into a Fourier transform of a time-dependent nuclear autocorrelation function can be advantageous, computationally and from the point of view of establishing contact with the pictorial aspect of the Franck-Condon principle.

The considerations above have implications for the traditional unimolecular reaction scheme where excitation and subsequent dissociation is (implicitly) separated. Thus, we have in the present paper considered the prerequisites for the definition of a unimolecular rate constant when the excitation is carried out with a continuous wave laser. Equation (41) constitute an expression for the rate constant when dissociation takes place via electronic predissociation. This equation is an exact dynamical expression for the rate-given that the coupling to the continuum is sufficiently weak. This means, in particular, that the laser is assumed to be in perfect resonance with a given (quasibound) state on the bound electronic surface and that these states decay independently (nonoverlapping resonances). Work concerning the relation between exact rate expressions and statistical microcanonical expressions for the rate ${ }^{23}$ is an interesting and active field of research. ${ }^{24,25}$

\section{ACKNOWLEDGMENTS}

This work was supported by the Danish Natural Science Research Council. One of us (N.E.H.) wish to thank J. A. Beswick for stimulating discussions.

${ }^{1}$ R. Schinke, Photodissociation Dynamics (Cambridge University, Cambridge, 1993).

${ }^{2}$ N. E. Henriksen, Adv. Chem. Phys. 91, 433 (1995).

${ }^{3}$ G. Herzberg, Spectra of Diatomic Molecules (Van Nostrand Reinhold, New York, 1950).

${ }^{4}$ E. J. Heller and R. C. Brown, J. Chem. Phys. 79, 3336 (1983).

${ }^{5}$ K. C. Kulander and E. J. Heller, J. Chem. Phys. 69, 2439 (1978).

${ }^{6}$ M. V. Rama Krishna and R. D. Coalson, Chem. Phys. 120, 327 (1988).

${ }^{7}$ E. J. Heller, R. L. Sundberg, and D. Tannor, J. Phys. Chem. 86, 1822 (1982).

${ }^{8}$ E. J. Heller, J. Chem. Phys. 68, 2066 (1978).

${ }^{9}$ E. J. Heller, Acc. Chem. Res. 14, 368 (1981)

${ }^{10}$ P. Villarreal, S. Miret-Artés, O. Roncero, G. Delgardo-Barrio, J. A. Beswick, N. Halberstadt, and R. D. Coalson, J. Chem. Phys. 94, 4230 (1991).

${ }^{11}$ A. D. Hammerich, A. Nitzan, and M. A. Ratner, Theor. Chim. Acta. 89, 383 (1994).

${ }^{12}$ M. Cho and R. J. Silbey, J. Chem. Phys. 103, 595 (1995).

${ }^{13}$ R. D. Coalson, Chem. Phys. Lett. 147, 208 (1988).

${ }^{14} \mathrm{R}$. Loudon, The Quantum Theory of Light, 2nd ed. (Oxford, New York, 1983).

${ }^{15}$ R. Kosloff, J. Chem. Phys. 92, 2087 (1988).

${ }^{16}$ N. E. Henriksen, Chem. Phys. Lett. 197, 620 (1992).

${ }^{17}$ R. L. Sundberg and E. J. Heller, Chem. Phys. Lett. 93, 586 (1983).

${ }^{18}$ A. Messiah, Quantum Mechanics (North-Holland, Amsterdam, 1961).

${ }^{19}$ R. L. Sundberg and E. J. Heller, J. Chem. Phys. 80, 3680 (1984).

${ }^{20} \mathrm{G}$. N. Watson, Theory of Bessel Functions, 2nd ed. (Cambridge University, Cambridge, 1952).

${ }^{21}$ J. Franck, Trans. Faraday Soc. 21, 536 (1925).

${ }^{22}$ E. U. Condon, Phys. Rev. 32, 858 (1928).

${ }^{23}$ J. C. Lorquet and B. Leyh-Nihant, J. Chem. Phys. 92, 4778 (1988).

${ }^{24}$ F. Remacle and R. D. Levine, J. Phys. Chem. 95, 7124 (1991).

${ }^{25}$ U. Peskin, H. Reisler, and W. H. Miller, J. Chem. Phys. 101, 9672 (1994). 